\title{
Track-Following Control with Active Vibration Damping of a PZT-Actuated Suspension Dual-Stage Servo System ${ }^{1}$
}

\author{
Yunfeng $\mathrm{Li}^{\dagger}$, Federico Marcassa ${ }^{\ddagger}$, Roberto Horowitz ${ }^{\dagger}$, Roberto Oboe ${ }^{\ddagger}$, and Robert Evans ${ }^{\S}$ \\ ${ }^{\dagger}$ Dept. of Mechanical Engineering, University of California at Berkeley, CA 94720-1740, USA \\ ${ }^{\ddagger}$ Dept. of Information Engineering, University of Padova, PD, 35131, ITALY \\ ${ }^{\S}$ Hutchinson Technology Incorporated, Hutchinson, MN 55350, USA
}

\begin{abstract}
This paper discusses the controller design of a PZTactuated suspension dual-stage servo system in hard disk drives. The proposed control structure includes an active vibration damping control loop and a trackfollowing control loop. The vibration damping control loop, which runs at a faster rate than the trackfollowing control loop, utilizes a PZT element on a PZT-actuated suspension as a vibration sensor to damp the resonance modes of the voice coil motor (VCM) and the PZT actuator. The vibration-damping controller is designed using Kalman filter based state feedback control techniques. A simple dual-stage track-following controller is designed, based on the damped actuator model, using the sensitivity function decoupling design method. Simulation and experimental results are presented to demonstrate the benefits of this control scheme in expanding servo control bandwidth and suppressing airflow excited structural vibrations.
\end{abstract}

\section{Introduction}

The use of a PZT-actuated suspensions as a second stage actuator has been proposed as one approach to dual-stage actuation in hard disk drives [1]. Compared to the actuated slider/head approach, the main disadvantage of this approach is that the PZT actuator is located between the E-block arm and the suspension, and can excite their vibration modes. Normally notch filters are utilized in disk drive servo control designs to ensure the stability of the control system. However, notch filters reduce phase margin and detrimentally affect the controller robustness [2], [3].

Furthermore, the arm and suspension vibration modes can be excited by the airflow generated from disk rotation, which causes undesirable head off-track mo-

\footnotetext{
${ }^{1}$ Research supported by the National Storage Industry Consortium (NSIC) and the Computer Mechanics Laboratory (CML) of U.C. Berkeley. Corresponding author E-mail: horowitz@me.berkeley.edu
}

tion. Airflow excited vibration (windage) is one of the major position error sources for high-RPM disk drives. In order to generate large motion range and reduce driving voltage, PZT-actuated suspensions are usually designed to have a relatively low frequency in-plane sway mode. This makes them to be more susceptible to airflow excitations than conventional suspensions.

A controller designed using notch filters cannot attenuate the windage effect on the structural vibration modes [3]. Moreover, because most of these vibration modes have resonance frequencies that are higher than the servo bandwidth, the windage-induced vibrations are often amplified by the closed-loop servo system. In [3], a phase-stabilized servo controller has been designed to suppress the track-mis-registration (TMR) due to airflow excited structural vibrations. Active vibration control is another approach to increase servo bandwidth and suppress vibration induced TMR [2],[4].

Because the sampling frequency of the position error signal (PES) is limited by the data storage efficiency, additional vibration sensors are usually needed to implement active vibration control. For a PZT-actuated suspension, one of the two PZT elements can be utilized as a vibration sensor to damp the head stack and suspension assembly vibration modes [4]. In this paper, a track-following control design combined with this vibration damping control scheme is presented. A new state feedback damping controller has been designed based on Kalman filter modal estimation. A simple track-following controller is designed using a sensitivity function decoupling method [5]. Notch filters are not used in the track-following control design because the vibration modes are adequately damped.

Section 2 describes the modelling of the control system. Section 3 discusses the damping controller and track-following controller designs. Section 4 presents the experimental results obtained with a hard disk drive (HDD) controlled using an external DSP and a Laser Doppler Vibrometer (LDV), which confirm the efficacy of the proposed control scheme in suppressing structural vibrations and enhancing the overall performance of the servo system. Section 5 concludes the paper. 

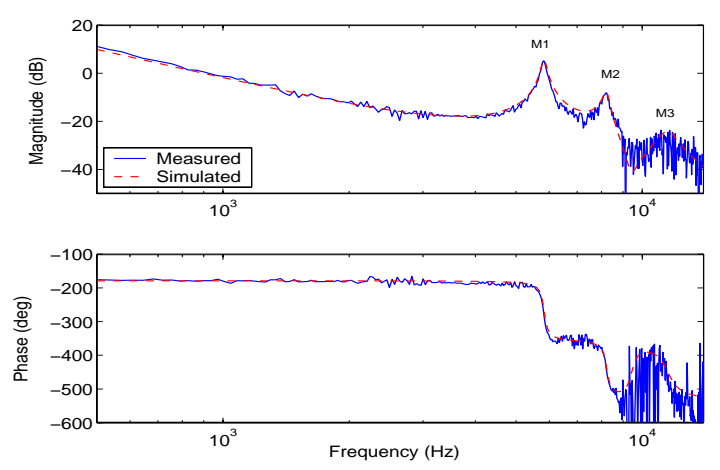

(a)
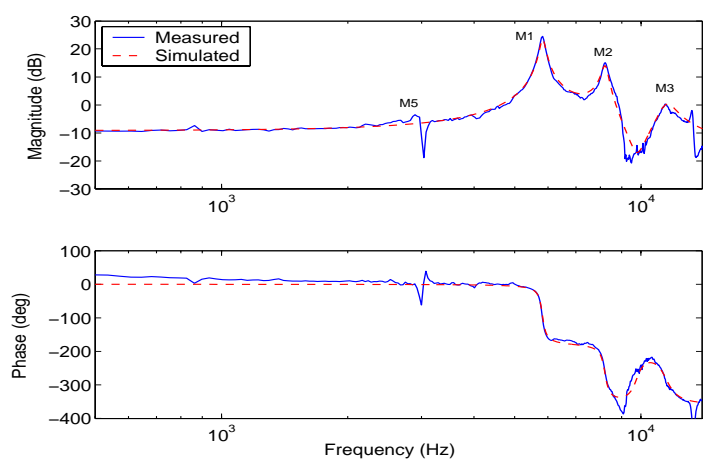

(c)
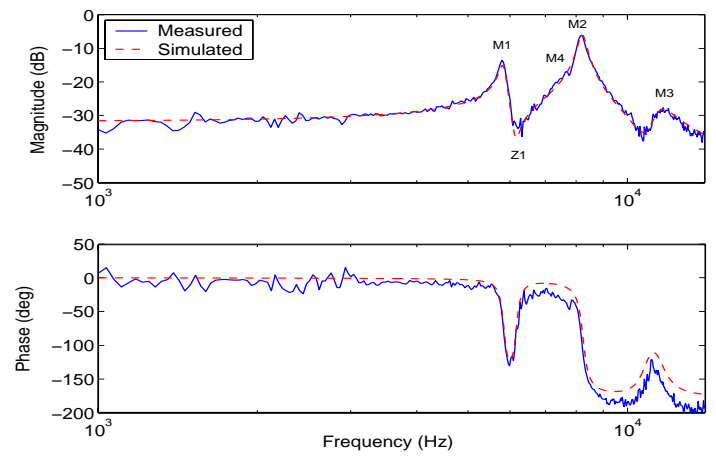

(b)
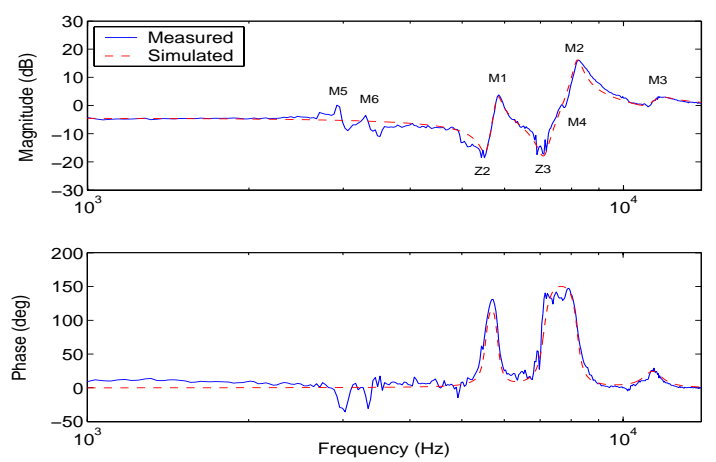

(d)

Figure 1: Frequency responses: (a) VCM input to head displacement; (b) PZT actuator input to head displacement; (c) VCM input to PZT sensor output ; (d) PZT actuator input to PZT sensor ouput

\section{Sensor and Actuator Modelling}

\subsection{Frequency Response Modal Testing}

The considered plant is a two-input two-output control system. Let $u_{1}$ and $u_{2}$ be the input to the voice coil motor (VCM) and the PZT actuator respectively, and $y_{1}$ and $y_{2}$ be the head displacement output in the radial off-track direction and the $\mathrm{PZT}$ vibration sensor output respectively. The transfer function from $u_{j}$ to $y_{i}, G_{i j}(s), i, j=1,2$, can be written as

$$
G_{i j}(s)=\frac{K_{0}^{i j}}{s^{2}}+\sum_{n=1}^{N} \frac{\omega_{n}^{2} K_{n}^{i j}}{s^{2}+2 \zeta_{n} \omega_{n} s+\omega_{n}^{2}}+d_{i j},
$$

which is the summation of the rigid body mode, a number of structural vibration modes and a direct feeding term from the input to the output. In Eq. (1), $K_{0}^{i j}$ is the gain of the rigid body mode, $N$ is the total number of vibration modes being considered, $\omega_{n}$ and $\zeta_{n}$ are the natural frequency and the damping ratio of mode $n$ respectively, $K_{n}^{i j}$ is the modal constant of mode $n$ from $u_{j}$ to $y_{i}$, and $d_{i j}$ is the direct feed through factor from $u_{j}$ to $y_{i}$.

Fig. 1 shows the following measured and modelled frequency responses: a) from VCM input $u_{1}$ to head displacement $y_{1}$; b) from $\mathrm{PZT}$ actuator input $u_{2}$ to head displacement $y_{1}$; c) from VCM input $u_{1}$ to PZT sensor output $y_{2} ; \mathrm{d}$ ) from PZT actuator input $u_{2}$ to PZT sensor output $y_{2}$. The head displacement is measured using an LDV.

The modal parameters, $\zeta_{n}, \omega_{n}, K_{n}^{i j}, n=1, \ldots, N$, $i, j=1,2$, in Eq. (1) have been extracted from the measured frequency responses, which are shown as solid lines in Fig. 1, using the peak-magnitude modal testing method [6]. The dashed lines are the simulated frequency responses of the identified model.

As shown in Fig. 1, the major vibration modes of the PZT-actuated suspension dual-stage actuator in our setup includes the VCM actuator butterfly mode (M1 in the figures), the suspension sway mode (M2), the suspension 2nd torsion mode (M3), and the suspension 1st torsion mode (M4). The butterfly mode is generated from the coupling of the in-plane sway mode of the E-block arm and the coil, in which the arm and the coil move out of phase with respect to each other around the pivot. The suspension sway mode is also called the PZT actuator actuation mode, or the motor mode.

As shown in Fig. 1a, the frequency response from VCM input $u_{1}$ to head displacement $y_{1}$ is dominated 
by the rigid body mode in the low frequency range and the structural vibration modes in the high frequency range. Fig. $1 \mathrm{~b}$ shows that the $\mathrm{PZT}$ actuator can excite the VCM actuator butterfly mode through the swage connection with the E-block arm. The coupling of the butterfly mode and the suspension sway mode results in a pair of lightly damped zeros (Z1 in the figure), which follow the poles of the butterfly resonance mode (M1).

From the frequency responses of the PZT sensor outputs in Fig. 1c-1d, it can be seen that the PZT sensor can pick up most of the off-track vibration modes of the head stack and suspension assembly when they are excited by the control inputs. The PZT sensor does not sense the rigid body mode, as expected. However, the PZT sensor picks up some non-off-track modes near 3 kHz (M5 and M6 in the Fig. 1c-1d), which have little effect on head off-track motion and will be modelled as plant noise modes, as discussed in section 2.3 and section 3.1 .

\subsection{State Space Model Realization}

The control system model can be represented using a state space model format based on the transfer functions in Eq. (1) and the extracted parameters. The rigid body mode is not observable from the PZT sensor output, and hence not included in the state space model. The PZT sensor is able to sense the vibration of modes M1, M2 and M3, but no M4. Modes M5, M6, and M7 have little contributions to the head off-track motion (see Figs. 1 and 2), therefore, they are not included in the control design model because they are either not controllable or difficult to control. A state space realization including the three vibration modes M1, M2, and M3 is:

$$
\begin{aligned}
& \dot{\mathbf{x}}=\mathbf{A x}+\mathbf{B u}, \\
& \mathbf{y}=\mathbf{C x}+\mathbf{D u},
\end{aligned}
$$

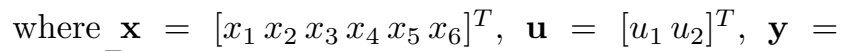
$\left[\begin{array}{ll}y_{1} & y_{2}\end{array}\right]^{T}$, and the decoupled state matrices can be written as

$$
\begin{array}{ll}
\mathbf{A}=\left[\begin{array}{ccc}
A_{1} & 0_{2 \times 2} & 0_{2 \times 2} \\
0_{2 \times 2} & A_{2} & 0_{2 \times 2} \\
0_{2 \times 2} & 0_{2 \times 2} & A_{3}
\end{array}\right], \quad \mathbf{B}=\left[\begin{array}{c}
B_{1} \\
B_{2} \\
B_{3}
\end{array}\right], \\
\mathbf{C}=\left[\begin{array}{cccccc}
c_{11} & 0 & c_{12} & 0 & c_{13} & 0 \\
c_{21} & 0 & c_{22} & 0 & c_{23} & 0
\end{array}\right], \quad \mathbf{D}=\left[\begin{array}{c}
0_{1 \times 2} \\
D_{2}
\end{array}\right],
\end{array}
$$

where each sub-matrix is expressed as

$$
\begin{aligned}
& A_{n}=\left[\begin{array}{cc}
0 & 1 \\
a_{n 1} & a_{n 2}
\end{array}\right], \quad B_{n}=\left[\begin{array}{cc}
0 & 0 \\
b_{n 1} & b_{n 2}
\end{array}\right] \\
& n=1,2,3 .
\end{aligned}
$$

The coefficients of matrices A are given by:

$$
a_{n 1}=-\omega_{n}^{2}, \quad a_{n 2}=-2 \zeta_{n} \omega_{n}, \quad n=1,2,3 .
$$

Finally, by normalizing the PZT sensor output equation with $c_{2 n}=1, n=1,2,3$, it results

$$
\begin{aligned}
& b_{n 1}=K_{n}^{21}, \quad b_{n 2}=K_{n}^{22}, \\
& c_{1 n}=K_{n}^{11} / K_{n}^{21}=K_{n}^{12} / K_{n}^{22}, \\
& n=1,2,3 .
\end{aligned}
$$

\subsection{Airflow Excited Vibrations}

Fig. 2 shows the power spectral densities (PSDs) of the head off-track motion (upper half) and the PZT sensor output (bottom half) when the spindle is rotating in a 7200-RPM disk drive and no control action is applied.
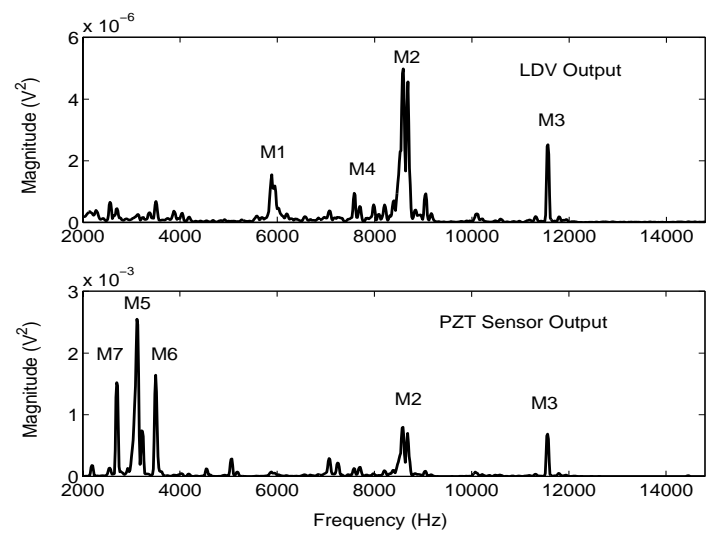

Figure 2: PSDs of the head off-track motion and the PZT sensor output due to airflow excited vibrations.

As shown in the figure, the major off-track modes excited by the airflow generated by disk rotation include the butterfly mode (M1), the suspension sway mode (M2), the suspension 2nd torsion mode (M3), and the suspension 1st torsion mode (M4). The PZT sensor is able to sense the vibrations of modes M2 and M3 when they are excited by airflow disturbances, but no M1 and M4. Modes M5, M6, and M7 have a large contribution to the PZT sensor output, yet they have little contributions to the head off-track motion. These modes are probably related to the bending modes of the suspension and they are excited by the airflow disturbances in the out-of-the-plane direction. They will be modelled as sensor noise modes in the Kalman filter design.

\section{Controller Design}

The proposed overall control structure, which includes a vibration-damping control loop and a trackfollowing control loop, is based on the block diagram shown in Fig. 3. $P$ represents the augmented plant model, which will be described in section 3.1.1, and 
w represents the airflow disturbances acting on the system. The damping controller is implemented using the PZT sensor output $y_{2}$, so that its sampling frequency will not be limited by that of the PES. A inner-loop vibration-damping controller is designed first. Then, the outer-loop track-following controller is designed based on the damped actuator model.

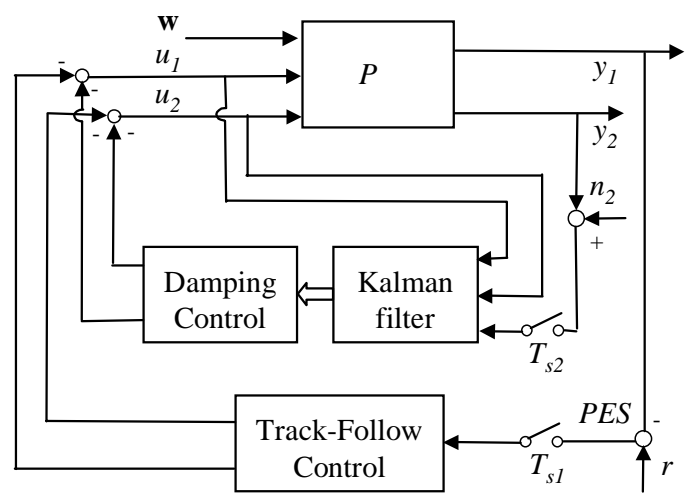

Figure 3: A block diagram of the control system.

\subsection{Damping Control Design}

In [4], a vibration-damping controller was designed using the $H_{\infty}$ control design method, in which weighting functions were used to characterize disturbance, noise and control design objectives. In order to have a better characterization of the sensor noise and to directly account for the computational time delay effect, a new damping control design method based on Kalman filter and state feedback control is proposed. An augmented plant model with disturbance and sensor noise modes has been used to design the Kalman filter for model estimation. The state feedback damping controller has been designed using the discrete-time-linearquadratic-regulator (DLQR) method.

\subsubsection{Augmented plant model for Kalman} filter design: As shown in Fig. 2, modes M5, M6, and M7 have a large contribution to the PZT sensor output when excited by airflow disturbances, but have little contribution to the head off-track motion. Moreover, they are either not controllable or weakly controllable by the control inputs. Thus, they will be modelled as sensor noise and will be accounted for in an augmented plant model described by

$$
\begin{aligned}
& {\left[\begin{array}{c}
\dot{\mathbf{x}} \\
\mathbf{x}_{\mathbf{w}}^{\cdot}
\end{array}\right]=\left[\begin{array}{cc}
\mathbf{A} & \mathbf{0} \\
\mathbf{0} & \mathbf{A}_{\mathbf{w}}
\end{array}\right]\left[\begin{array}{c}
\mathbf{x} \\
\mathbf{x}_{\mathbf{w}}
\end{array}\right]+\left[\begin{array}{cc}
\mathbf{B} & \mathbf{0} \\
\mathbf{0} & \mathbf{B}_{\mathbf{w}}
\end{array}\right]\left[\begin{array}{c}
\mathbf{u} \\
\mathbf{w}
\end{array}\right],} \\
& {\left[\begin{array}{l}
\mathbf{y}_{\mathbf{1}} \\
\mathbf{y}_{\mathbf{2}}
\end{array}\right]=\left[\begin{array}{cc}
\mathbf{C}_{1} & \mathbf{0} \\
\mathbf{C}_{\mathbf{2}} & \mathbf{C}_{\mathbf{w}}
\end{array}\right]\left[\begin{array}{c}
\mathbf{x} \\
\mathbf{x}_{\mathbf{w}}
\end{array}\right]+\left[\begin{array}{ll}
\mathbf{D} & \mathbf{0}
\end{array}\right]\left[\begin{array}{c}
\mathbf{u} \\
\mathbf{w}
\end{array}\right],}
\end{aligned}
$$

where $\mathbf{x} \in \mathbb{R}^{6}$ represents the state of the three vibration modes to be controlled (M1, M2, M3); A, B, $\mathbf{C}_{\mathbf{1}}, \mathbf{C}_{\mathbf{2}}$, $\mathbf{D}$ are described by Eq. $(2-6) ; \mathbf{x}_{\mathbf{w}} \in \mathbb{R}^{6}$ represents the state of the three noise modes (M5, M6, M7); w $\in \mathbb{R}^{6}$ is a fictitious white disturbance vector which is used to characterize the excitation to each vibration mode by airflow disturbances; $\mathbf{A}_{\mathbf{w}}$ was estimated from the measured PSD in Fig. 2; $\mathbf{B}_{\mathbf{w}}$ and $\mathbf{C}_{\mathbf{w}}$ are normalized.

The discrete time model of $P$ in Eq. (7), with computational time delay $t_{d}$, can be written as follows

$$
\begin{aligned}
& {\left[\begin{array}{c}
\mathbf{x}(k+1) \\
\mathbf{x}_{\mathbf{w}}(k+1) \\
\mathbf{u}(k)
\end{array}\right]=\left[\begin{array}{ccc}
\mathbf{\Phi} & 0 & \boldsymbol{\Gamma}_{\mathbf{1}} \\
0 & \boldsymbol{\Phi}_{\mathbf{w}} & 0 \\
0 & 0 & 0
\end{array}\right]\left[\begin{array}{c}
\mathbf{x}(k) \\
\mathbf{x}_{\mathbf{w}}(k) \\
\mathbf{u}(k-1)
\end{array}\right]+} \\
& +\left[\begin{array}{c}
\boldsymbol{\Gamma}_{\mathbf{2}} \\
0 \\
I
\end{array}\right] \mathbf{u}(k)+\left[\boldsymbol{\Gamma}_{\mathbf{w}}\right] \mathbf{w}(k) \\
& {\left[\begin{array}{l}
\mathbf{y}_{\mathbf{1}}(k) \\
\mathbf{y}_{\mathbf{2}}(k)
\end{array}\right]=\left[\begin{array}{lll}
\mathbf{C} & \mathbf{C}_{\mathbf{w}} & \mathbf{D}
\end{array}\right]\left[\begin{array}{c}
\mathbf{x}(k) \\
\mathbf{x}_{\mathbf{w}}(k) \\
\mathbf{u}(k-1)
\end{array}\right]}
\end{aligned}
$$

Assuming the computational time delay $t_{d}$ is smaller than the sampling time $T_{s 2}$, we have the following results

$$
\begin{aligned}
\Phi & =e^{A T_{s}}, \Phi_{w}=e^{A_{w} T_{s}} \\
\Gamma_{1} & =\int_{0}^{t_{d}} e^{A \tau} B d \tau \\
\Gamma_{2} & =\int_{t_{d}}^{T_{s}} e^{A \tau} B d \tau
\end{aligned}
$$

3.1.2 Kalman filter and DLQR design: A discrete-time Kalman filter with prediction and correction steps was designed based on the augmented discrete-time plant model defined in Eq. (8), to estimate the state of the vibration modes. The two design parameters of the Kalman filter are the covariance matrix $\mathbf{W}$ of the disturbance vector $\mathbf{w}$ and noise variance $V_{2}$ of the PZT sensor output $y_{2}$. The disturbance covariance matrix $\mathbf{W}$ can be estimated from the PSD of the PZT sensor output (see Fig. 2), since the disturbance input matrix $\mathbf{B}_{\mathbf{w}}$ and the output matrix $\mathbf{C}_{\mathbf{w}}$ are normalized. The measurement noise covariance $V_{2}$ can be used as a design parameter to set the bandwidth of the observer.

The design of the feedback gain matrix was based on the discretization with computational delay $t_{d}$ of the control plant model defined in Eq. (2). The feedback damping controller gain $\mathbf{K}$ was generated by a DLQR which minimizes

$$
\sum_{k}\left\{y_{1}^{2}(k)+\mathbf{u}^{T}(k) \mathbf{R u}(k)\right\},
$$

where $y_{1}$ is the head displacement output. The design parameter in the DLQR design is the control action weight matrix $\mathbf{R}$, which can be tuned to obtain the desired system responses. 

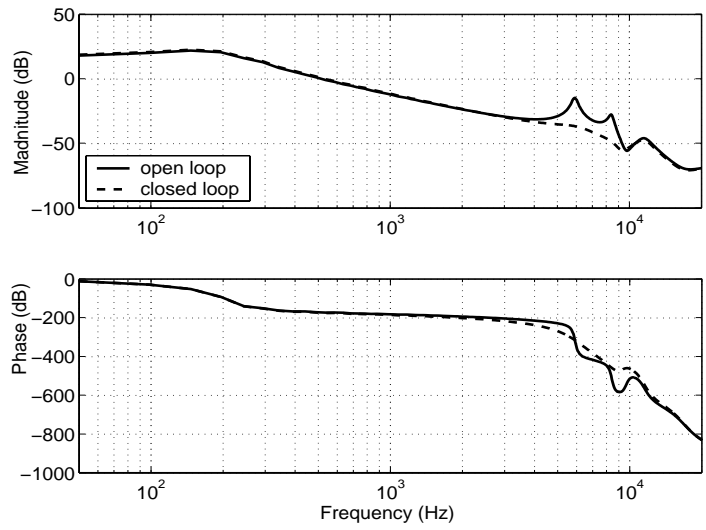

(a)
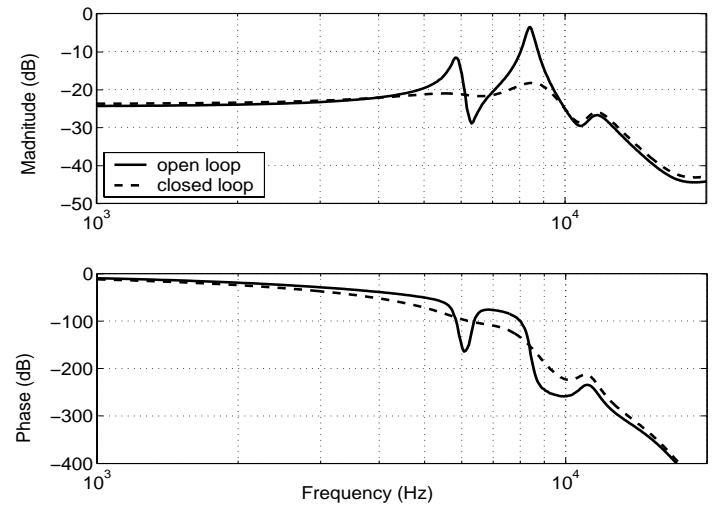

(b)

Figure 4: Simulated frequency responses: (a) VCM input to head displacement; (b) PZT actuator input to head displacement.

3.1.3 Simulation results: As briefly described in the previous section, the choice of the process noise weights was based on the PSD of the open-loop PZT sensor output, the remaining parameters, the measurement noise variance $V_{2}$ and the control action weight matrix $\mathbf{R}$ can be set/tuned in order to obtain the desired damped system. A Simulink model was used to tune these parameters, which also allowed us to test the effects of the computational delay and the noise induced by the single floating point arithmetic on the damping controller. Fig. 4 shows simulation results of the damped transfer functions from VCM and PZT actuator to head displacement, respectively.

\subsection{Track-Following Controller Design}

Once the inner-loop damping control design is completed, the outer-loop track-following controller can be designed based on the damped actuator models, which have frequency responses shown in Fig. 4. There are several popular techniques for designing dual-stage track-following controllers. In this paper, a simple sensitivity transfer functions decoupling method, which is also referred as the decoupled master-slave, or the series compensator method, has been used [5], [7].

Fig. 5 shows a block diagram for dual-stage trackfollowing controller design using this method. In the figure, $r$ represents the track runout; $G_{V C M}$ and $G_{M A}$ are the (damped) VCM and PZT actuator models, respectively. $K_{V C M}$ and $K_{M A}$ are the VCM and PZTactuator loop controllers, respectively. The displacement of the PZT actuator relative to that of the VCM, $R P E S S$, is estimated by multiplying the PZT control input by the DC gain of the PZT actuator, $g_{P Z T}$. It can be shown that the total closed-loop sensitivity function of the dual-stage servo system in Fig. 5 is approx-

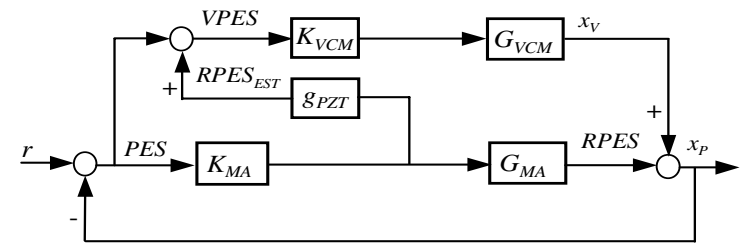

Figure 5: Block diagram of the track-following controller design

imately equal to the series of the sensitivity functions corresponding to the VCM and PZT-actuator loops. Thus, the design of the dual-stage servo controller can be decoupled. First, the VCM loop compensator can be designed independently. Then, the PZT loop compensator is designed to expand the bandwidth of the overall control system [5].

The VCM loop compensator, $K_{V C M}(s)$, was designed to be a lead-lag compensator. The VCM loop gain cross-over frequency was chosen to be $800 \mathrm{~Hz}$. The PZT actuator loop compensator, $K_{M A}(s)$, was designed to be a lag compensator. The PZT actuator loop gain cross-over frequency was chosen to be 2500 $\mathrm{Hz}$. Notch filters are not used because the resonance modes are adequately damped by the damping control loop.

The Bode plot of the overall open-loop transfer function with the track-following controller from $r$ to $y_{1}$ (damping control loop closed) is shown as the solid lines in Fig. 6. The gain cross over frequency, the gain margin and the phase margin of the dual-stage control system are $2425 \mathrm{~Hz}, 3.4 \mathrm{~dB}$ and $37^{\circ}$, respectively. As a comparison, the open-loop Bode plot of a similar track-following controller design without utilizing damping control, but with notch filters, is shown as the dashed lines in Fig. 6. The gain cross over frequency, 

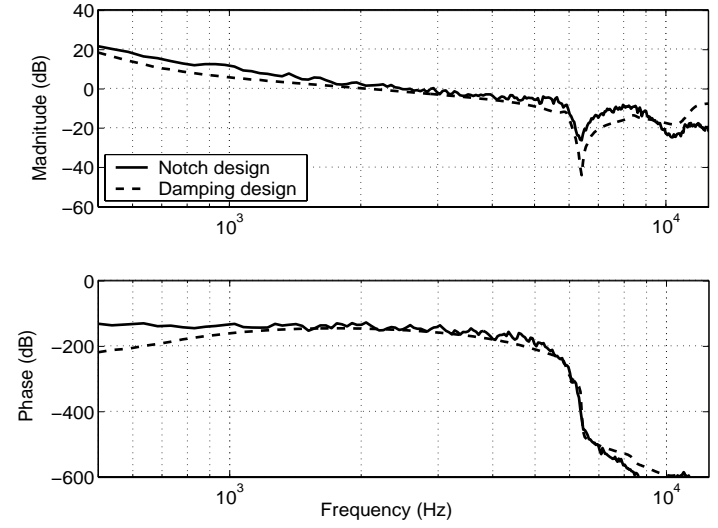

Figure 6: Open-loop Bode plots

the gain margin and the phase margin are $2150 \mathrm{~Hz}, 5 \mathrm{~dB}$ and $33^{\circ}$, respectively. Using damping control, a higher bandwidth and larger phase margin were achieved for the track-following control system.

\section{Experimental Results}

The HDD used in the experimental testing has a spindle rotation speed of 7200 RPM. The head off-track motion was measured using an LDV with a resolution of $0.5 \mu \mathrm{m} / \mathrm{V}$. The control algorithms were implemented using a Texas Instrument TMS320C6711 floating point DSP. The sampling frequency used for the damping controller was $50 \mathrm{kHz}$, while that for the track-following controller was $25 \mathrm{kHz}$.

At first, the inner-loop damping controller was tested. Fig. 7 compares the frequency responses of the damped system to those of the open-loop system, from VCM and PZT actuator to head displacement, respectively. Both the butterfly mode and the suspension sway mode are attenuated by the damping controller.

Secondly, the combined outer-loop track-following controller and the inner-loop damping controller was tested. The PZT loop lag compensator zero were tuned in order to obtain the best result from the point view of both standard deviation and flat PSD of the position error.

In order to validate and check the effectiveness of this control scheme, the experimental results were compared to the results obtained using notch filters in the trackfollowing controller instead of the inner-loop damping control. Fig. 8 shows the FFT of the PES for the two designs. As shown in the figure, using the proposed damping controller, the high frequency vibration can be greatly attenuated. The track-following controller with damping control also has a larger disturbance attenuation in the low frequency range because of the increased bandwidth. The standard deviation of the position error of the damping control design is $4.8 \mathrm{~nm}$, while that of the traditional notch filter design is 6.1 $\mathrm{nm}$. Note that because the head position is measured by an LDV in the experimental testing, these results do not reflect the effects on the TMR due to track runout.

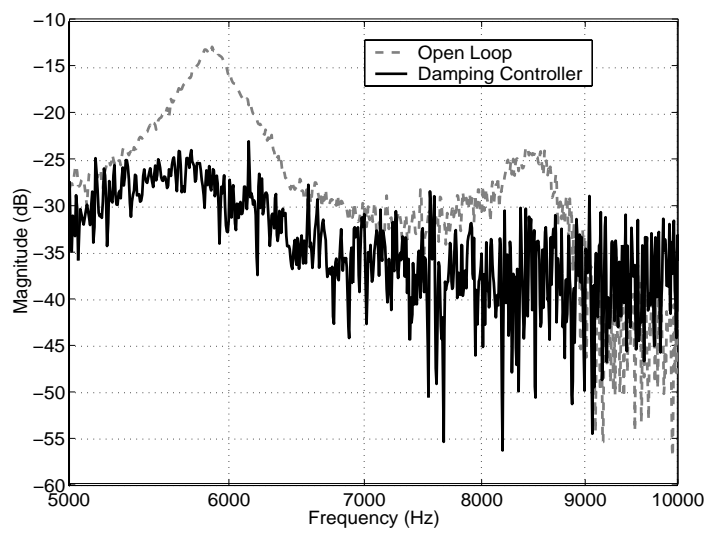

(a)

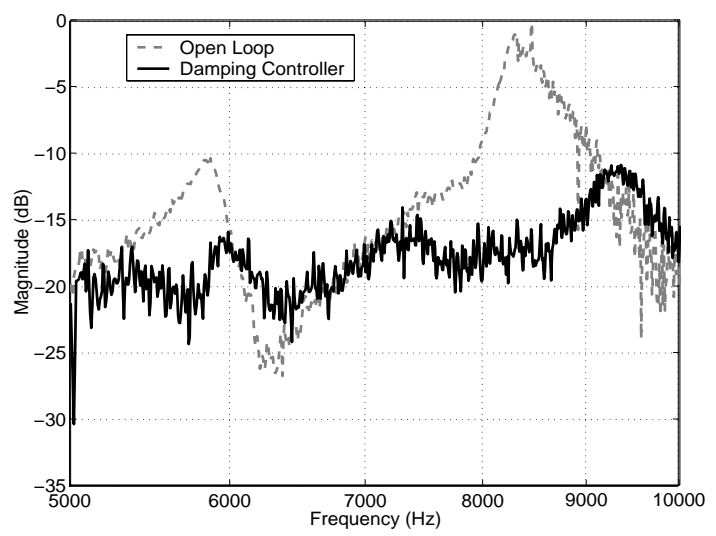

(b)

Figure 7: Measured frequency responses: (a) VCM input to head displacement; (b) PZT actuator input to head displacement.

Fig. 9 shows the simulated and identified sensitivity function using the track-following controller combined with the inner-loop damping controller. The sensitivity $0 \mathrm{~dB}$ cross over frequency is about $1.5 \mathrm{kHz}$. Fig. 10 shows the PES in the time domain.

\section{Conclusion}

A track-following controller design with active vibration damping has been proposed for a PZT-actuated suspension dual-stage servo system. A state feedback inner-loop damping controller was designed to actively damp multiple resonances modes using one PZT ele- 


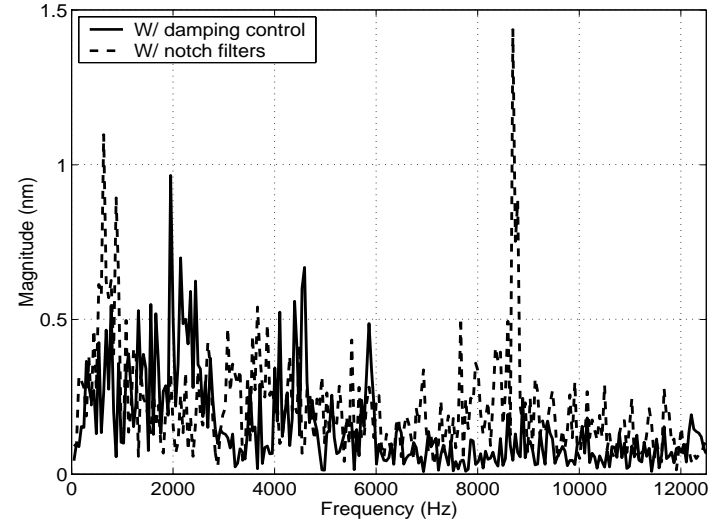

Figure 8: FFT of the head off-track motion (PES)

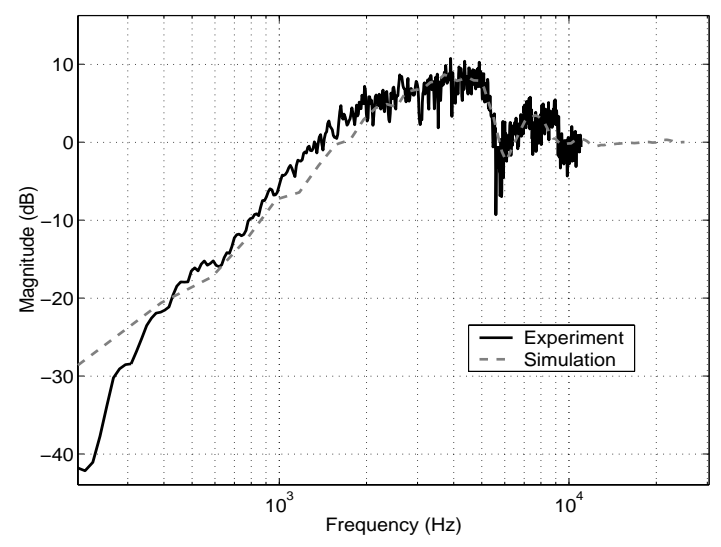

Figure 9: Sensitivity function Bode plot.

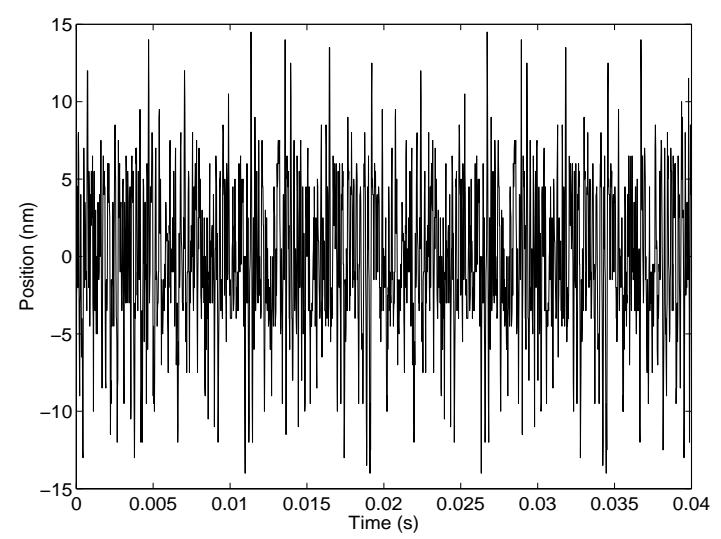

Figure 10: Head off-track motion (PES) ments on a PZT-actuated suspension as a vibration sensor. A high bandwidth outer-loop track-following controller was designed based on the damped dual-stage actuator model. Experimental results confirm the effectiveness of the proposed control scheme in suppressing structural vibrations and enhancing the overall performance of the servo system.

The use of one PZT element as a vibration sensor provides a simple and effective solution to active vibration control of a PZT-actuated suspension dual-stage servo system. It can be used to damp not only the suspension sway mode, but also the head-stack assembly butterfly mode. However, there are also some limitations in this scheme since the PZT element was not originally designed to be a sensor. It cannot sense some off-track modes, while it picking up some non-off-track modes. This makes the vibration controller to be complicated. The vibration control design could be greatly simplified if optimally designed vibration sensors are available. The research in the design and fabrication of such sensors is now being conducted by our research group.

\section{References}

[1] R.B. Evans, J.S. Griesbach, and W.C. Messner "Piezoelectric microactuator for dual-stage control", IEEE Transaction on Magnetics, pp 977-81, Vol. 35, March, 1999

[2] F.-Y. Huang, T. Semba, W. Imaino, and F. Lee, "Active damping in HDD actuator" IEEE Trans. Magn., vol. 37, pp. 847-849, Mar. 2001.

[3] M. Kobayashi, S. Nakagawa, and S. Nakamura "A phase-stabilzed servo controller for dual-stage actuators in hard disk drives", IEEE Transaction on Magnetics, March, 2003

[4] Y. Li, R. Horowitz, and R. Evans "Vibration Control of a PZT Actuated Suspension Dual-Stage Servo System using a PZT Sensor", IEEE Transaction on Magnetics, March, 2003

[5] Y. Li and R. Horowitz, "Design and Testing of Track-Following Controllers for Dual-Stage Servo Systems with PZT Actuated Suspensions", Microsystem Technologies, Vol. 8, Issue 2-3, May 2002.

[6] D.J. Ewins, Modal Testing: Theory, Pratice and Application, 2nd edition, Research Studies Press Ltd., 2000

[7] K. Mori, T. Munemoto, H. Otsuki, Y. Yamaguchi, and K. Akagi, "A dual-stage magnetic disk drive actuator using a piezoelectric device for a high track density," IEEE Transactions on Magnetics, vol. 27, no. 6, pp. 5298-5300, November 1991. 